\title{
24 Die multiethnische und multireligiöse Krim unter zarischer Herrschaft: Die tatarische Bevölkerung - Geschlechterverhältnisse
}

\begin{abstract}
I now proceed to give some account separately of other colonists [...]. The Nogay Tatars, if not the original inhabitants of the country, at least wandered over the immense steppes of New Russia, with their tents, flocks, and herds, at the time of the Russian Conquest, and the ages before [...]. The government has at length conquered the inveterate prejudices of this wandering horde, and induced them to lay aside their roving habits, settling them in villages, and inciting them to cultivate the ground. They are, however, of all the colonists, far the worst cultivators. ${ }^{1}$
\end{abstract}

Diese Beschreibung der Nogaier stammt von der Engländerin Mary Holderness, deren Leben vor allen Dingen deshalb Spuren hinterlassen hat, da sie über ihren (vermutlich) zwischen 1816 und 1820 dauernden Krimaufenthalt einen mehrmals aufgelegten Reisebericht verfasst hat: Gemeinsam mit ihrem Mann und ihren Kindern verbrachte sie einige Jahre als landwirtschaftliche Kolonistin auf der Krim. Die Familie war damit der Aufforderung des anglikanischen Geistlichen Arthur Young (1769-1827) gefolgt, der sich seit 1805 wiederholt auf Einladung der russländischen Regierung zu agrarischen Studien in Russland aufgehalten hatte und 1810 in der Nähe des ehemaligen Kefe (Feodosija) ein großes Gut erworben hatte; nur deshalb - und anders als sein berühmterer Vater gleichen Namens (1741-1820), der sich als Agrarwissenschaftler und Publizist einen Namen gemacht hatte - sei er überhaupt einer Studie wert, wie sein eigener Biograph wenig wohlwollend schrieb. ${ }^{2}$ In jedem Fall verdanken wir Youngs Engagement einen Bericht über die Krim, der aus mehreren Gründen interessant ist: Er wurde von einer Frau verfasst, was erst einmal im Kontext der Reisejournale über die Halbinsel an sich nicht exzeptionell ist, denken wir beispielsweise an die so bekannte Beschreibung der Lady Craven im Vorfeld der Taurischen Reise (vgl. Kapitel 23). ${ }^{3}$ Hervorzuheben ist aber der vergleichsweise lange Aufenthalt Holderness', der dieser eine eingehendere Befassung mit dem Locus und der Bewohnerschaft ermöglichte als es bei ihrer Landsmännin Craven der Fall war. Holderness selbst betonte in ihrem Vorwort, dass trotz „zahlloser Mängel“ („numberless deficiencies“) ihr langer Aufenthalt und ihr weibliches Geschlecht

1 Holderness (1823); Anonyma (1855), 140 f. Vgl. zur ihrer Biographie Mary Holderness (2017).

2 Gazley (1956), 360.

$3 \mathrm{Zu}$ reisenden Frauen im imperialen Kontext vgl. z. B. Marbo (1991), 163; Pratt (1992). Speziell über die Krim Jobst (2001d).

Ә OpenAccess. (c) 2020 Kerstin S. Jobst, publiziert von De Gruyter. (cc))BY Dieses Werk ist lizenziert unter der Creative Commons Attribution 4.0 International. https://doi.org/10.1515/9783110520620-026 
bei der Abfassung des Berichts von Vorteil gewesen seien: „I am induced to think that, as a resident and a female, I possessed advantages for acquiring information, superior to those of the passing traveller." ${ }^{4}$ Doch wie in zahllosen anderen europäischen Reiseberichten der Zeit nicht nur über die Krim, sondern allgemein über vermeintlich exotische Gegenden, so waren die Urteile Angehöriger einer selbsterklärten zivilisierten Nation über fremde Kulturen und Nationalitäten von einem Überlegenheitsgefühl geprägt: Die russische Krim-Bevölkerung, obzwar den neuen Machthabern zugehörig, galt ihr durchweg als ungebildet, sofern sie nicht hochadlig war. ${ }^{5}$ Schlimmer noch kamen die Nogaier - wie bereits in älteren Berichten - weg. Sie seien miserable Landwirte, aber immerhin so versierte Pferdediebe, dass selbst die Kosaken nicht an sie herankämen, wie Holderness ironisch vermerkte. Ihr Gesamturteil über die nicht-sesshaften BewohnerInnen der nördlichen Krim war ein schlechtes: „The moral character of the Nogays is of the worst description, and there is hardly any kind of mischief which they will not perpetrate. " ${ }^{6}$ Ein weiteres Mal zeigt sich die Geringschätzung nomadisch lebender Gemeinschaften, die seit der Antike (vgl. Kapitel 3) ein Signum vieler Krim-Berichte gewesen ist; vielleicht geschah diese scharfe Abgrenzung in zivilisiert versus unzivilisiert auch in Rücksicht auf den erwarteten Publikumsgeschmack, denn es existierte ein gewisser Widerspruch zu den Beschreibungen über MuslimInnen, die in einem administrativen Kontext entstanden sind: So galt für die zarische Verwaltung, dass diese bis zum Krimkrieg eher pragmatisch/analytisch über ihre neuen UntertanInnen berichtete und sich normativer Äußerungen meist enthielt, obwohl sie ihnen grundsätzlich misstraute. ${ }^{7}$ Eine Unterhaltung und Spannung erwartende Leserschaft wünschte freilich eine weniger nüchterne Schilderung des Fremden in den europäischen Peripherien - und dieser Erwartung kamen Autorinnen und Autoren häufig nach.

Der von Holderness selbst ins Spiel gebrachte Vorteil ihres Geschlechts griff vor allen Dingen dann, wenn sie über eine Sphäre schrieb, die männlichen Autoren in der Tat verschlossen blieb: nämlich die der tatarischen Frauen, hielt sich doch die weitgehende Segregation weiblicher und männlicher Lebensbereiche in den krimtatarischen Zentren wie Bağçasaray oder Qarasuvbazar bis in die ersten Jahrzehnte des 20. Jahrhunderts. Somit sind Berichte aus erster (männlicher) Hand über muslimische Frauen selten bzw. eher Phantasieprodukte. ${ }^{8}$ Während das Urteil Holderness' über die männlichen Tataren der Südküste mehr oder

\footnotetext{
4 Holderness (1823), 2.

5 Holderness (1823), z. B. 122 f.

6 Holderness (1823), 141.

7 Dies ist eine Beobachtung der Verfasserin dieser Zeilen. Vgl. auch Fisher (1978), 82.

8 Jobst (2007b), 209.
} 
weniger genauso stereotyp ausfiel wie über die Nogaier, gelangen ihr interessante Beschreibungen von Mutter-Kind-Beziehungen oder tatarischen Stillgewohnheiten. ${ }^{9}$ Anders bewertete sie die Geschlechterverhältnisse in der muslimischen Gesellschaft im Allgemeinen: Für die meisten westeuropäischen Autorinnen war der imaginierte Orient ein Ort weiblichen Sklaventums und häufig auch eine Sphäre, wo das Ausleben ungezügelter Leidenschaften vermutet wurde, wobei der Topos des Harems bzw. die (auf der Krim aus wirtschaftlichen Gründen allerdings nur eingeschränkt praktizierte) Vielehe als Beweis genommen wurde. Aber große Prüderie galt ebenfalls als typisch orientalisch. ${ }^{10}$ Holderness' Einschätzung bildete keine Ausnahme:

A Tatar wife is most completely the slave of her husband, and that the men consider her such, I had from the mouth of one of the most respectable of them. Thus she is only desirable as she serves to gratify his passions, or to connect him with some Tatar of better family or greater riches than himself. ${ }^{11}$

Allein bei Bauernfamilien meinte sie eine größere auch emotionale Nähe zwischen den Paaren zu erkennen, welche wahre Liebe nicht ausschloss. ${ }^{12}$ Parallelen zu den Geschlechterverhältnissen in der eigenen Kultur und Gesellschaft etwa hinsichtlich der weit verbreiteten ökonomisch-politisch motivierten (Oberschichts-)Heiraten ignorierte sie genauso wie die in christlichen und muslimischen Kulturen existierenden weiblichen Handlungsspielräume.

Inwieweit die zarische Herrschaft über die Krim Einfluss auf die Geschlechterverhältnisse innerhalb der muslimischen Gemeinschaften nahm, kann auf der Grundlage der gegenwärtigen Forschung nicht beurteilt werden. Unstrittig ist Folgendes: Keineswegs alle Musliminnen auf der Krim waren verschleiert bzw. vollverschleiert. ${ }^{13}$ Heiraten zwischen Angehörigen des Islams und des Christentums unabhängig von der Konfession waren in zarischer Zeit eine Ausnahme ${ }^{14}$, waren aber von der russischen Administration durchaus erwünscht. Rassismus spielte im Vergleich zu westeuropäischen Kolonialreichen eine geringere Rolle, und russische Akteure versprachen sich von muslimisch-christlichen Verbin-

9 Holderness (1823), vgl. u. a. $223 \mathrm{f}$.

10 Melman (1992), 52.

11 Holderness (1823), 225.

12 Holderness (1823), 225.

13 Dazu Jobst (2007b), 208-212. Vgl. auch einige Abbildungen in dem kommentierten Wiederabdruck eines erstmalig 1856 und 1857 erschienenen Werkes des Naturkundlers und Mitglieds der Petersburger Akademie Gustav Ferdinand Richard Radde (1831-1903): Radde (2008).

14 Williams (2001), 124, will keinen dokumentierten Fall kennen, anders bei Jobst (2007b), 216. 
dungen eine Annäherung der ,fremden` Kulturen an die slavisch-orthodoxe Bevölkerungsmehrheit. ${ }^{15}$

Insgesamt waren die Auswirkungen der Annexion von 1783 für die ehemalige Titularnation ganz unabhängig vom Geschlecht gewaltig. Der Verlust der (eingeschränkten) Selbstständigkeit des frühneuzeitlichen Chanats und die unfreiwillige Eingliederung in ein Reich der ,Ungläubigen', d.h.von NichtmuslimInnen, waren bereits tiefgreifende Zäsuren. Besonders nachhaltig waren aber die Folgen für die Bevölkerungsentwicklung, Wirtschaft, Kultur und die allgemeinen Partizipationsmöglichkeiten. ${ }^{16}$

Wie schon erwähnt, setzte St. Petersburg in seiner kolonialen Politik gegenüber dem ehemaligen Chanat auf die Inklusion der neuen UntertanInnen, auch um die Gefahr einer starken pro-osmanischen Opposition zu verhindern, welche die neuerliche Verbindung mit der Hohen Pforte anstreben würde. ${ }^{17}$ Ein entsprechendes Anreizsystem sollte dabei helfen: Neben der bereits erwähnten Kooption tatarischer Mirza gehörte die muslimische Geistlichkeit (Ulema) zu den Profiteuren der russischen Herrschaft, weshalb sie auch den Romanovs bis zum Verfall des Zarenreichs treu ergeben war. Die traditionellen Rechte der Geistlichkeit auf der Halbinsel wurden weitgehend anerkannt. Für den russischen Staat lag der Vorteil dieser Politik des ,Teile und Herrsche' darin, dass die islamische Gelehrtenschaft und der im Verlauf der ersten Jahrzehnte nach der russischen Annexion von der Lokalpolitik weitgehend (außer in Bağçasaray und in Qarasuvbazar) ausgeschlossene Adel unterschiedliche Interessen entwickelten und keine gemeinsame Front gegen die neue Macht entstehen konnte. ${ }^{18}$,Geboten wurde der Ulema ein großes Maß an Selbstkoordination: Einer im Jahr 1794 formierten muslimischen „Geistlichen Versammlung“ stand ein von der Zarin bzw. dem Zaren ausgewählter Mufti vor. Dieser erhielt, wie im Zarenreich üblich, einen Adelstitel und war für die religiösen Belange der MuslimInnen der Krim und derjenigen in der Provinz bzw. dem Gouvernement Taurien verantwortlich. ${ }^{19}$ Der Mufti und die übrigen muslimischen Geistlichen wurden vom Staat entlohnt, womit ein besonderes Loyalitätsverhältnis zur Krone begründet wurde. Der Besitz der muslimischen Gemeinschaft - in erster Linie, aber nicht ausschließlich von Grund und Boden - wurde von staatlichen Abgaben befreit. ${ }^{20}$ Daneben behielt die

15 Jobst, u.a. (2008), 45.

16 Im Folgenden orientiere ich mich u. a. an Jobst (2014); Jobst (2017a).

$17 \mathrm{Zu}$ diesem Kalkül vgl. z. B. den zarischen Historiker Laškov (1886), 91.

18 Fisher (1979), 84-85.

19 Die 1784 gebildete Tavričeskaja oblast' (Taurische Provinz) wurde 1802 zur Tavričeskaja gubernija (Taurisches Gouvernement).

20 Djuličev (2002), 241. 
muslimische Gemeinschaft die Kontrolle über das vakıf-Stiftungsvermögen. ${ }^{21}$ Die religiösen und rituellen Angelegenheiten und verwaltungsrelevante Aufgaben wie das Führen von Geburts- und Sterberegistern blieben ebenfalls eine Domäne der Ulema. ${ }^{22}$

Neben diese älteren Ordnungs- und Verwaltungsmuster traten die neuen, aus der Metropole eingeführten: Die Parallelität tradierter und neu implementierter Rechts- und anderer Systeme ist in imperialen Kontexten zumindest für eine Übergangszeit üblich. ${ }^{23}$ Im Falle der Krim erhoffte sich St. Petersburg aber - wie bereits dargelegt - mittel- bis langfristig die weitestmögliche strukturelle Angleichung an die zentralrussischen Gebiete. Zudem wünschte man sich - u.a. durch gesetzlich-administrative Maßnahmen befördert - einen idealerweise durch die orthodoxe Taufe abgeschlossenen Akkulturationsprozess der nichtslavischen, nicht-christlichen Krimbevölkerung. Dies war im Übrigen ein niemals erreichtes Ziel, denn weder auf der Krim noch in den anderen muslimischen Gebieten des Imperiums kam es zu nennenswerten Konversionsbewegungen auf freiwilliger Basis. In einer Hinsicht unterschieden sich die Verhältnisse auf der Halbinsel aber von anderen, ebenfalls stark muslimisch geprägten Regionen, wie beispielsweise an der Wolga: Während es in den bereits in der Mitte des 16. Jahrhunderts eroberten ehemaligen Chanaten von Kazan' und Astrachan' phasenweise zu einer massiven orthodoxen Missionstätigkeit gekommen war, die nicht selten in gewaltsamen Zwangskonversionen gipfelte, war diese auf der Krim bereits von Katharina II. verboten worden. ${ }^{24}$ Akteure der orthodoxen Kirche bemühten sich zwar seit den 1840er Jahren zunehmend, das orthodoxe Christentum auf der zu diesem Zeitpunkt noch überwiegend muslimischen Halbinsel zu stärken und eine orthodoxe Infrastruktur durch den (Wieder-)Aufbau von Kirchen und Klöstern zu schaffen, indem sie auf die Krim als Ort ,der Taufe der Rus“ rekurrierten (vgl. Kapitel 2). Auch strebten kirchliche Kreise danach, mit Hilfe archäologischer Grabungen zu beweisen, dass die Krim seit dem 3. Jahrhundert durchgehend ein vom Christentum und Slaven geprägter Ort gewesen sei. Allerdings waren die Resultate eher unbefriedigend: Der Missionierung der dortigen

21 Dies bezeichnet fromme Stiftungen islamischen Rechts. Muslimische Gläubige unterstützen damit religiöse Institutionen (z. B. Moscheen, Medresen oder Mausoleen) oder auch allgemeine wichtige Einrichtungen einer Gemeinschaft (z. B. Brunnen): Hartung (2005).

22 Lazzerini (1988), $131 \mathrm{f}$.

23 Zum Russländischen Reich unter Einbeziehung der allgemeinen Imperiumsgeschichte vgl. Burbank (2006). Ein weiteres Beispiel an der Westgrenze des Imperiums schildert Ganzenmüller (2013).

24 Vgl. hierzu u.a. Lemercier-Quelquejay (1967). Eine Betrachtung der sog. Apostasiewelle im 19. Jahrhundert bietet Frings (2010). 
nicht-orthodoxen BewohnerInnen wurden durch St. Petersburg und zum Teil auch durch die örtliche Verwaltung enge Grenzen gesetzt. Nur unter den außerhalb der orthodoxen Kirche stehenden christlichen Gruppen wie den Altgläubigen, KatholikInnen (z. B. polnischstämmige Grundbesitzer) oder ProtestantInnen (z.B. aus den Reihen der deutschen KolonistInnen) durfte ohne Beschränkungen missioniert werden. Selbst nach dem Krimkrieg, als in der russischsprachigen Öffentlichkeit die Halbinsel kollektiv in einen heiligen ,russischen' Raum umgedeutet wurde (vgl. Kapitel 26), galten diese von Katharina II. festgesetzten Regeln noch. Immerhin konnte 1860 eine eigene orthodoxe Diözese eingerichtet werden. ${ }^{25}$

Über die Kooption krimtatarischer weltlicher Oberschichten in das russländische Rangsystem wurde bereits berichtet. Dies förderte mittelbar die Akkulturation der Mirza, die zum Teil den Lebensstil des russischen Adels und die russische Sprache für sich entdeckten und sich vereinzelt sogar orthodox taufen ließen. ${ }^{26}$ Dies traf auf Mitglieder des in der Chanatszeit so einflussreichen Clans der Şirin zu, welche erkannt hatten, dass die Übernahme des orthodoxen Christentums Voraussetzung für die Erlangung politischen/sozialen Einflusses war. Einzelne Angehörige der sich nun einer russifizierten Namensform bedienenden und seit 1836 gefürsteten Familie Širinskij (= Şirin) erlangten im Russländischen Imperium hohes Ansehen und wichtige Positionen. Platon A. Širinskij-Šichmatov (1790-1853) beispielsweise gelang eine besonders eindrucksvolle Karriere: Als Absolvent der Kaiserlichen Marine-Akademie nahm er u.a. am Vaterländischen Krieg 1812 bis 1814 gegen Frankreich teil. Nach verschiedenen Positionen im Ministerium für Volksaufklärung (Ministerstvo narodnogo prosveščenija) wurde er ab 1850 schließlich dessen Minister. Er war zudem Mitglied der Akademie der Wissenschaften, wo er u.a. für die Abfassung von Wörterbüchern des Altkirchenslavischen und der russischen Sprache mitverantwortlich zeichnete. Überdies wirkte er als Schriftsteller. ${ }^{27}$ Ein Aufstieg in der Hierarchie des Reiches war also auch für nicht-slavische, ,fremde‘ Untertanen möglich, wenn ihnen denn die Seite der Macht das Prädikat der (sozialen) Ebenbürtigkeit zuerkannte und die ,Fremden' sich in Sprache, Religion und Habitus der metropolitanen Kultur annäherten. Im Vergleich mit dem britischen Imperium waren solche ,Erfolgsgeschichten` im russländischen Reich weitaus häufiger. ${ }^{28}$

Für die muslimische Bauernschaft bedeutete die Eingliederung in das Imperium eine nachhaltige Zäsur, die auf den ersten Blick allerdings nicht unbedingt

25 Dazu Kozelsky (2010).

26 Bennigsen (1972), 147.

27 Rudakov (1903).

28 Vgl. Jobst, u. a. (2008), 45. 
zu erkennen war: Sie blieben weiterhin persönlich frei, da die bis 1861 in den zentralrussischen Gebieten geltende Leibeigenschaft in den neueroberten Gebieten des nördlichen Schwarzen Meeres nicht eingeführt wurde. Während beispielsweise im Gouvernement Kiew 1857 über sechzig Prozent der Bauernschaft leibeigen waren, waren es auf der Krim nur gut sechs Prozent. Dabei handelte es sich nicht um muslimische Untertanen, sondern zumeist um Slaven (nach heutiger Diktion primär um RussInnen und UkrainerInnen), die an der Seite ihrer Grundherren aus den nördlichen Gebieten zugewandert waren. ${ }^{29}$ Dessen ungeachtet ist eine deutliche Verschlechterung der Lage der krimtatarischen Bauernschaft zu konstatieren, u. a. da russische, aber auch tatarische Großgrundbesitzer sich konstant an der Beschneidung traditioneller bäuerlicher Rechte (z. B. der Wasser- oder Wiesennutzung) versuchten und den Anteil Leibeigener auf der Krim deutlich erhöhen wollten. Die Grundbesitzer argumentierten unabhängig von ihrer Herkunft mit mangelnder Leistungsfähigkeit der eingewurzelten Bauern oder interessanterweise mit der (vermeintlichen) Notwendigkeit der Vereinheitlichung des Reiches: Man müsse die Bauernschaft auf der Halbinsel so stellen wie in den zentralrussischen Gebieten, was praktisch die Überführung tatarischer Bauern in die Leibeigenschaft bedeutet hätte. Die zarische Administration verschloss sich diesen adligen Wünschen, insbesondere während der Amtszeit des General-Gouverneurs von Neurussland (1823-1844), Michail S. Voroncov (17821856). ${ }^{30}$

Innerhalb des Imperiums waren die krimtatarischen BewohnerInnen de jure keine Gruppe minderen Rechts im Vergleich mit den BewohnerInnen der zentralrussischen Gebiete, zumal sie persönlich frei blieben. Im Vergleich zu den übrigen MuslimInnen im Zarenreich ist Folgendes zu konstatieren: Die Regierungszeit Katharinas II. bedeutete insgesamt für die MuslimInnen eine Zäsur, da die vordem herrschende gezielte Unterdrückungspolitik etwa gegenüber den MuslimInnen im Wolga-Ural-Gebiet eingestellt wurde. Islamische Institutionen wurden nach einer Phase forcierter Integrationspolitik in der ersten Hälfte des 18. Jahrhunderts reinstalliert, eine kulturelle Renaissance des Islams war zu vermerken, und Mullahs konnten als niedrige Amtsträger fungieren. Ein relevanter Unterschied zur Krim bestand darin, dass es im Wolga-Ural-Gebiet selten institutionalisierte Stiftungsverwaltungen gab, und dass es in der Mitte des 19. Jahrhunderts in der Wolga-Ural-Region nur noch Relikte eines staatlicherseits anerkannten Adels gegeben hat. ${ }^{31}$ Somit ist von einer vergleichsweise bevorzug-

29 Herlihy (1986), $79 \mathrm{f}$.

30 Rhinelander (1990), 89f.

31 Ich folge hier Noack (2000), besonders 49-77. 
ten Lage der Krim-MuslimInnen auszugehen. Dennoch ist die zarische, wie später übrigens auch die sowjetische Politik (vgl. Kapitel 32) gegenüber den muslimischen BewohnerInnen der Halbinsel von einer „Diskrepanz zwischen (rechtlichen) Normsetzungen und (gelebten) Praktiken“ gekennzeichnet. Diese konnte „der krimtatarischen Bevölkerung situativ zum Vor- oder aber auch zum Nachteil gereichen.“32 Für die zarische Zeit gilt: Nicht allein Elitenangehörige wie aus dem Geschlecht der Širinskij-Šichmatovs konnten von den neuen Verhältnissen profitieren, sondern auch sozial weniger exponierte Aufsteiger. Für einen Großteil der bäuerlichen Krimtatarinnen und Krimtataren traf dies trotz ihres persönlich freien Status nicht zu. Am deutlichsten lässt sich dies am krimtatarischen Exodus in das Osmanische Reich zeigen, der von Brian G. Williams ausführlich untersucht worden und eines der signifikantesten Phänomene dieser ersten ,russischen Phase“ in der Geschichte der Krim ist. Analytisch, in der Art der Durchführung und in Hinsicht auf die Motivlage der verschiedenen Akteure ist diese Migrationsbewegung von „sürgün“ - der Deportation im Mai 1944 (vgl. Kapitel 34) - zu trennen. Dies erschließt sich freilich nicht auf den ersten Blick: Grundsätzlich waren Bevölkerungsverschiebungen zwischen christlichen und muslimischen Imperien zwischen dem ausgehenden 18. und dem beginnenden 20. Jahrhundert keine Seltenheit. Vergleichbare Migrationsbewegungen gab es beispielsweise in der Kaukasus-Region, auf der unter habsburgischer Herrschaft stehenden BalkanHalbinsel, im nördlichen Afrika, dessen Gebiete unter französische Herrschaft gefallen waren, oder im Herrschaftsgebiet des Sultans. Was waren die Hintergründe? In den Islamwissenschaften wird häufig auf das Gebot hingewiesen, nach dem eine jede Muslima, ein jeder Muslim die Verpflichtung habe, aus dem Herrschaftsgebiet der NichtmuslimInnen auszuwandern. Die muslimische Gemeinschaft - die sog. Umma - sollte dadurch geschützt werden. Diese religiös motivierte Auswanderung (hijra; hiğra) mag bei vielen Migrationen dieser Zeit eine Rolle gespielt haben, von einer regelrechten Pflicht zur Auswanderung aus dem Land der Ungläubigen zu sprechen, geht aber vielen IslamwissenschaftlerInnen zu weit. ${ }^{33}$ Im Fall der Halbinsel kam eine Reihe von Motiven zusammen, welche die Migrationswellen mittelbar veranlassten: Neben den hier nicht im Einzelnen zu klärenden religiösen Gründen stürzten tiefgreifende ökonomische Veränderungen inklusive dem Eindringen kapitalistischer Produktionsbedingungen die krimtatarische Agrargesellschaft in eine Krise, was Auswanderungen Vorschub leistete. ${ }^{34}$ In der Land- und Viehwirtschaft der Halbinsel waren noch im

32 Jobst (2017a), 93.

33 Dazu Meier F. (1991); Masud (1990).

34 Karpat (1984/1985) sowie Karpat (1986). 
19. Jahrhundert ca. 90 Prozent der Bevölkerung beschäftigt. Dies ist eine für die Zeit nicht ungewöhnliche Quote, doch ist verständlich, dass Probleme in diesem Bereich Auswirkungen auf die Gesamtökonomie hatten. Konflikte mit Grundbesitzern, der Anbau von für das Klima ungeeigneten Produkten durch zugewanderter Landwirte oder auch der zum Teil von russischen Besatzern verursachte Wassermangel verschärften die Lage der Bauernschaft. ${ }^{35}$ Die allgemeine politische Lage und die Haltung imperialer AkteurInnen gegenüber den MuslimInnen kamen hinzu: Die unmittelbar im Zusammenhang mit der Annexion von 1783 geäußerte Meinung Potemkins, dass die Krim ohne die Tataren eine bessere sei, teilten nicht wenige Elitenangehörige auch noch in späteren Dekaden. Insbesondere nach dem Krimkrieg war das Misstrauen gegenüber dem religiös Fremden so spürbar, dass Gerüchte - als „Information im Medium des Hörensagens ohne bestimmbaren Autor" ${ }^{\text {*36 }}$ - viele krimtatarische Familien zur Ausreise veranlassten. Edward Lazzerini fasst die Stimmungslage folgendermaßen zusammen: „[E]ndless rumormongering [...] fed the anxieties of many Tatars, thereby contributing significantly to the episodic, and sometimes frenzied, flight abroad. "37 Marc Blochs Beobachtung, dass Gerüchte schon Massen bewegt hätten, zeigte im krimtatarischen Fall drastische Konsequenzen. ${ }^{38}$

35 Lynch (1965), 162. Insbesondere der im letzten Viertel des 19. Jahrhunderts zu Weltruhm gelangte Wein und Sekt von der Krim war anfänglich kein Erfolg, da die von auswärtigen Winzern auf die Halbinsel gebrachten prestigeträchtigen Trauben keine guten Erträge brachten.

36 Neubauer H.-J. (1998), 13.

37 Lazzerini (1997), hier 170.

38 Bloch (1963), hier 43. 
